\title{
Gesundheitsregionen: Der Mensch im Mittelpunkt, nicht Krankheiten
}

Thomas Wernlia, Urs Zanoni ${ }^{b}$

a Direktor pflegimuri

${ }^{b} \mathrm{MPH}$, Departement Gesundheit und Soziales Kanton Aargau

Unser Versorgungssystem fokussiert auf Krankheiten, Medizin und den Akutbereich; ausserdem sind die Leistungserbringer mangelhaft vernetzt. Darunter leiden die Behandlungsqualität und die Patientensicherheit, namentlich bei chronisch kranken Menschen. Ein mögliches Rezept dagegen sind Gesundheitsregionen: Die Versorgungspartner bilden gemeinsam eine Trägerschaft, verpflichten sich zu verstärkter Zusammenarbeit, beziehen die ganze Bevölkerung ein und auch den Sozialbereich - ein Denkanstoss aus dem Aargau.

Das Schweizer Gesundheitssystem ist den Herausforderungen der Zukunft nur bedingt gewachsen: Es ist zu stark auf die kurative Medizin ausgerichtet und vernachlässigt die psychosozialen Bedürfnisse der Patienten sowie die Gesundheitsförderung und Prävention. Ebenso ist es zu stark auf die Akutversorgung (in Spitälern) ausgerichtet und vernachlässigt die dauerhafte Betreuung von chronisch kranken, häufig dementen Menschen sowie die Koordination zwischen den Leistungserbringern. Begünstigt werden diese Defizite durch das Krankenversicherungsgesetz, das mit den

\section{Gesundes Freiamt 2025}

Salome (77) und Herbert Habsburger (74) nehmen seit 2019 über ihren Krankenversicherer am Versorgungsmodell Gesundes Freiamt teil. Insgesamt bestehen Verträge mit 12 Krankenversicherern, die 90 Prozent der Menschen den Zugang zu diesem Modell ermöglichen. Salome und Herbert Habsburger sind auch Mitglied der Genossenschaft Gesundes Freiamt. Sie haben einmalig 500 Franken bezahlt und profitieren im Gegenzug von Vergünstigungen bei Kursen, Vorträgen, Ausflügen etc.

Die Habsburgers nutzen seit fünf Jahren, angeleitet durch ihren Enkel, das elektronische Patientendossier - wie fast die Hälfte der Menschen im Gesunden Freiamt.

Beim letzten Arztbesuch sprach Salome Habsburger ausführlich mit ihrem Hausarzt über die Chancen und Risiken eines neuen Medikaments. Das Gespräch war Teil des Programms "Gemeinsam für Ihre Gesundheit», das Patienten systematisch in Therapieentscheide einbezieht.

Herbert Habsburger nutzt seit drei Jahren eine Smartwatch und erfasst damit die Strecken, die er zu Fuss und mit dem Velo zurücklegt. Im Gegenzug erhält er bei PartnerFirmen des Gesunden Freiamts einen Rabatt.

Die älteste Tochter der Habsburgers besucht bei der Gesundheitsbildung Freiamt einen Kurs für pflegende Angehörige, weil sie ihre Eltern darin unterstützen will, möglichst lange zu Hause zu leben.

Per Newsletter erfahren die Habsburgers, dass 48 Firmen und Gemeindeverwaltungen mit 1600 Mitarbeitenden im Forum Betriebliches Gesundheitsmanagement Freiamt vereint sind.

Im Newsletter lesen Sie ausserdem, dass der Schweizerische Gemeindeverband das Freiämter Integrations-Programm «Sprache und Arbeit bauen Brücken» ausgezeichnet hat. veränderten Anforderungen an die Versorgung nicht Schritt hält.

Anderseits bestehen Möglichkeiten, das System zu verbessern, allen voran mit dem elektronischen Patientendossier (das entsprechende Bundesgesetz tritt im ersten Halbjahr 2017 in Kraft). Die Versicherten und Patienten können dann die eigenen Daten, die an den verschiedenen Behandlungsorten elektronisch erfasst sind, einsehen und allen beteiligten Fachpersonen zugänglich machen. Diese Transparenz wird die Qualität, Effizienz und Sicherheit der Behandlung und Betreuung massgeblich steigern, ebenso das Zusammenspiel der Fachpersonen und ihrer Einrichtungen.

\section{Patientensicht und Eigenverantwortung}

Die wichtigste Voraussetzung, um Fachpersonen besser zu vernetzen und die heutigen Koordinationsdefizite zu reduzieren, ist gegenseitiges Vertrauen. Dieses Vertrauen wiederum ist in aller Regel dort am grössten, wo sich Menschen persönlich kennen: in der Region. Der Aargau ist der Kanton der Regionen und verfügt mit den Regionalplanungsverbänden über Räume, die sich ausgezeichnet für ein zukunftsfähiges Versorgungsmodell eignen. Zudem ist der Aargau in der elektronischen Vernetzung weit fortgeschritten, ebenso in der Vorbereitung auf das elektronische Patientendossier [1].

Aus diesen Überlegungen entstand die Absicht, eine erste Gesundheitsregion zu etablieren: Am 30. März trafen sich in Muri gut 30 Personen von Versorgungspartnern und diskutierten erstmals die Vision «Gesundes Freiamt» (Abb. 1): 
- Bevölkerungsorientiert: Die Versorgung ist auf die gesamte Bevölkerung ausgerichtet, umfasst also Gesunde, Risikogruppen und Kranke jeden Alters.

- Wohnortnah: Die Angebote sind so gestaltet und mit elektronischen Hilfsmitteln unterstützt, dass die Behandlung und Betreuung möglichst lange in der Region erfolgen kann, insbesondere bei Patienten zu Hause (Home Care; Ambient Assisted Living).

- Konsequente Vernetzung: Die Fachpersonen und ihre Einrichtungen arbeiten sektorenübergreifend, systematisch und verbindlich zusammen. Die einzelnen Behandlungs- und Betreuungsschritte sind optimal aufeinander abgestimmt und werden mit elektronischen Hilfsmitteln unterstützt (eHealth).

- Bedarfsgerechte Angebote: Die Betreuung von ausgewählten Personengruppen erfolgt bedarfs- und bedürfnisgerecht. Das sind vor allem chronisch kranke Menschen; fremdsprachige Menschen, die sich hier nur schlecht verständigen können; Erwerbstätige in belasteten Situationen.

- Medizinisches und Soziales Hand in Hand: Soziale und (fallweise) juristische Aspekte sind in die Betreuung einbezogen - und damit auch die Sozialdienste der Gemeinden, ebenso Fachorganisationen für Alter, Behinderung, Integration, Sucht sowie Gesundheitsligen, deren Tätigkeit vor allem auf die psychosozialen Bedürfnisse der Menschen ausgerichtet ist.

- Stärkung der Prävention: Die Versorgung bzw. Unterstützung der Menschen setzt so früh wie möglich ein; Gesundheitsförderung und Prävention (inkl. Früherkennung von Krankheiten) sind wichtige Teile des Modells.

- Stärkung der Patienten(-perspektive): Die Fachpersonen nehmen regelmässig die Sicht der Patienten ein

Bevölkerung im Freiamt

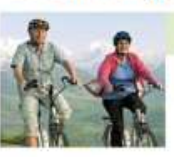

Gesunde (Primărprävention)

Risikogruppen (Sekundärprâvention)

Patienten (Tertiärprävention) Selbstsorge Ambulant

\section{Ambulant,}

Reha, Wiedereingliederung

\section{Langzeitpflege} ambulant \& stationăr

Palliative Begleitung

Gesundheitsförderung, Prävention, Behandlung, Betreuung, Begleitung

Trägerschaft

- Medizinische, pflegerische, soziale/juristische Leistungserbringer

- Vertretung von Versicherten/Patienten/Angehörigen/Vertrauenspersonen

- Regionalplanungsverband/Gemeinden/Kanton

- Krankenversicherer

Patientenorientiert, Koordiniert, Interprofessionell, Bedarfsgerecht, Wohnortnah (oder ihrer Angehörigen), damit die Betreuung möglichst schonend und effizient erfolgt. Patienten wiederum sind befähigt und motiviert, die eigenen Ansprüche an die Versorgung zu reflektieren und eigenverantwortlich zu handeln.

- Breit abgestützte Trägerschaft: Die Versorgungspartner der Region (inkl. Regionalplanungsverband/Gemeinden) bilden eine Trägerschaft und verpflichten sich auf gemeinsame Ziele und Leitsätze für die Zusammenarbeit. Die Trägerschaft kann spezielle Verträge mit Versicherern abschliessen für Leistungen, die im heutigen System kaum abgedeckt sind.

Bevölkerungs-orientierte Modelle haben sich bereits in mehreren Ländern etabliert (z.B. Holland, Deutschland [2], Österreich, Grossbritannien, USA). Auch in der Schweiz gibt es erste Gesundheitsregionen, zum Beispiel das Unterengadin [3] oder im Kanton Waadt [4], weitere - wie im Kanton St. Gallen und Laufental - sind in Vorbereitung. Es bestehen also genügend Erfahrungen und Erkenntnisse sowie erprobte Prozesse und Instrumente, die bei Bedarf zu nutzen sind.

\section{Versicherten- und Patientenrat}

Das Gesunde Freiamt (www.gesundes-freiamt.ch) soll der Bevölkerung, den Leistungserbringern sowie der ganzen Region zu Gute kommen. Das Modell erhöht die Sicherheit und Qualität der Behandlung und Betreuung, weil die Leistungserbringer besser miteinander vernetzt sind. Dies gilt besonders bei hochaltrigen, oft dementen Menschen, die neben medizinisch-pflegerischen Bedürfnissen meist auch soziale (Bewältigung des Alltags) und juristische haben (Erwachsenenschutz). Der starke Fokus auf Information und Beratung will die Bevölkerung überdies zu mehr Eigenverantwortung befähigen, wozu auch die Anwendung des elektronischen Patientendossiers gehört.

Die Leistungserbringer sollen eine ausgeprägte «Kultur des Gemeinsamen» entwickeln: Die einzelnen Berufsgruppen anerkennen die Kompetenzen der anderen und sind offen für neue Aufgabenteilungen. Das kann personelle Engpässe vermeiden und die Effizienz der Versorgung erhöhen. Für die Koordination von Menschen in komplexen Situationen werden speziell ausgebildete Fachleute und besondere IT-Lösungen eingesetzt. Da die sektorenübergreifenden Behandlungs- und Betreuungsprozesse bestmöglich organisiert sind, ist der einzelne Patient zu jeder Zeit am richtigen Ort. Dadurch würde die Infrastruktur der Leistungsanbieter optimal ausgelastet.

Fachleute und Bevölkerung sollen sich in hohem Masse mit dem Gesunden Freiamt identifizieren: Die Fach- 
personen bevorzugen Arbeitgeber in der Region, die Patienten wollen wenn immer möglich im Freiamt behandelt und betreut werden. Wenn der Regionalplanungsverband in der Trägerschaft vertreten ist, erleichtert das die Versorgungsplanung. Zudem würden Versorgungslücken oder -probleme rasch(er) erkannt und behoben. Die Bedürfnisse der Menschen könnte ein Versicherten- und Patientenrat in die Planung einbringen.

\section{Den Spielraum des KVG nutzen}

Das Gesunde Freiamt soll schrittweise entstehen inhaltlich wie geographisch. Angestrebt wird eine Trägerschaft, in der alle namhaften Partner vertreten sind: ambulante und stationäre Leistungserbringer, Gesundheitsligen und Fachorganisationen, Versicherte und Patienten, der Regionalplanungsverband und Gemeinden, der Krankenversicherer - und der Kanton, der das Vorhaben als Dienstleister unterstützt.

Die IG Gesundes Freiamt wäre zu Beginn mit minimalem organisatorischem und administrativem Aufwand tätig. Ihre möglichen Aufgaben: Sie initiiert Projekte, welche die Interessen sowie personellen und finanziellen Möglichkeiten ihrer Mitglieder bestmöglich berücksichtigen, unterstützt die Projektverantwortlichen und informiert alle Partner und die Bevölkerung.

Denkbar ist, die Trägerschaft später in eine Betriebsgesellschaft umzuwandeln (z.B. Verein, Genossenschaft, $\mathrm{GmbH})$. Eine solche Betriebsgesellschaft könnte spezielle Verträge mit Krankenversicherern abschliessen, die auch neue Vergütungsformen enthalten. Zumal das Krankenversicherungsgesetz hier beträchtlichen Spielraum bietet, namentlich bei Pauschalen.

Ausserdem ist zu prüfen, ob Versicherer bereit sind, ein spezielles Versicherungsmodell für das Gesunde Freiamt anzubieten. Die Bevölkerung wiederum könnte sich über eine Genossenschaft oder einen Verein an der Vision beteiligen (siehe "Gesundes Freiamt 2025»). Fazit: Regionale, bevölkerungsorientierte Versorgungsmodelle wie das Gesunde Freiamt dürften den Herausforderungen der Zukunft weit besser gewachsen sein als das heutige System, weil sie

- Gesunde, Risikogruppen und Patienten erfassen;

- die sektorenübergreifende, interprofessionelle $\mathrm{Zu}$ sammenarbeit fördern;

- das Medizinisch-Pflegerische mit dem Sozialen und Juristischen vernetzen (auch elektronisch);
- die Patientenperspektive und Eigenverantwortung unterstützen;

- die Versorgungspartner der Region in einer Trägerschaft vereinen.

Am 17. August 2016 führt das aargauische Gesundheitsdepartement zusammen mit den Regionalplanungsverbänden eine Fachtagung zu Gesundheitsregionen durch. Mehr Informationen dazu finden sich auf www. ag.ch/dgs $\rightarrow$ Über uns $\rightarrow$ Projekt \& Dossiers $\rightarrow$ MIVAG17.

\section{Bildnachweis \\ rrenoir.com}

Literatur

1 siehe www.ehealth-aargau.ch

2 Das Gesunde Kinzigtal besteht seit 2005 (www.gesundes-kinzigtal.de)

3 Gesundheitszentrum Unterengadin (www.cseb.ch)

4 Réseaux Santé Vaud (www.reseaux-sante-vaud.ch), gestützt auf das kantonale Gesetz für Versorgungsnetze

\section{Flächendeckende Koordination}

Dr. Markus Dieth, Präsident Baden Regio

Auch im Regionalplanungsverband Baden Regio steht die Gesundheitsversorgung im Fokus: In den 26 Gemeinden bieten neben stationären Einrichtungen sowie Haus- und Fachärzten rund 60 ambulant tätige Organisationen Unterstützung bei pflege- und betreuungsbedürftigen Menschen an. Die Angebote sind für die Betroffenen aber oft nur schwer zu unterscheiden.

Es besteht also die Gefahr, dass sich die Ratsuchenden nicht an die für ihre Bedürfnisse geeignetste Organisation wenden oder sogar keinen Zugang finden. Diese Gefahr ist umso grösser, je komplexer die Bedürfnisse sind oder wenn mehrere Fachpersonen den Unterstützungsbedarf nur gemeinsam abdecken können.

Vor diesem Hintergrund wurde, zusammen mit dem Institut für Pflege der Zürcher Hochschule für Angewandte Wissenschaften (Prof. Dr. Lorenz Imhof), das Projekt «Pflegerische Anlaufund Beratungsstelle Baden Regio" (PABS) gestartet. Dessen Hauptziel ist die Begleitung von Menschen in medizinischpflegerischen Fragen sowie die Vernetzung und Koordination der Angebote innerhalb von Baden Regio.

Angesichts der stetig steigenden Gesundheitskosten ist es besonders wichtig, dass Betroffene rasch eine bedürfnis- und situationsgerechte Unterstützung erhalten. Deshalb braucht es eine Stelle, welche die Komplexität erkennt, Kontakte vermittelt, berät und «die Fäden zusammenhält» - vor allem bei älteren Menschen, die möglichst lange selbständig bleiben möchten.

Eine PABS Baden Regio sollte aber Ratsuchenden jeden Alters (inkl. Angehörigen) zur Seite stehen. Sie ist keine Konkurrenz zu den etablierten Leistungserbringern, sondern will diese für die jeweilige Person optimal vernetzen und koordinieren. Aus der Überzeugung, dass die Förderung des Selbstmanagements einen effektiven Mehrwert schafft. 